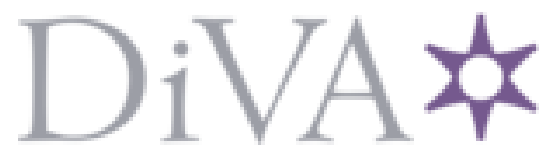

http://www.diva-portal.org

This is the published version of a paper presented at Intelligent Vehicles Symposium (IV), 2016 IEEE.

Citation for the original published paper:

Ward, E., Folkesson, J. (2016)

Vehicle localization with low cost radar sensors.

In: Intelligent Vehicles Symposium (IV), 2016 IEEE Institute of Electrical and Electronics

Engineers (IEEE)

http://dx.doi.org/10.1109/IVS.2016.7535489

N.B. When citing this work, cite the original published paper.

Permanent link to this version:

http://urn.kb.se/resolve?urn=urn:nbn:se:kth:diva-192860 


\title{
Vehicle localization with low cost radar sensors
}

\author{
Erik Ward ${ }^{1}$ and John Folkesson ${ }^{1}$
}

\begin{abstract}
Autonomous vehicles rely on GPS aided by motion sensors to localize globally within the road network. However, not all driving surfaces have satellite visibility. Therefore, it is important to augment these systems with localization based on environmental sensing such as cameras, lidar and radar in order to increase reliability and robustness. In this work we look at using radar for localization. Radar sensors are available in compact format devices well suited to automotive applications. Past work on localization using radar in automotive applications has been based on careful sensor modeling and Sequential Monte Carlo, (Particle) filtering. In this work we investigate the use of the Iterative Closest Point, $I C P$, algorithm together with an Extended Kalman filter, $E K F$, for localizing a vehicle equipped with automotive grade radars. Experiments using data acquired on public roads shows that this computationally simpler approach yields sufficiently accurate results on par with more complex methods.
\end{abstract}

\section{INTRODUCTION}

Localization is a fundamental requirement for autonomous driving vehicles. We propose a method for localizing an autonomous vehicle using solid state mono pulse short range radars, SRR. The method is based on Iterative Closest Point $I C P$, matching of current scans from the vehicles radars to scans from a previous traversal of the same road. These ICP matches are used as pose measurements in an Extended Kalman Filter, $E K F$, that then smooths these estimates using a vehicle motion model. The method is similar to the method in [1]. The novel aspect here is the use of the SRR radar and the evaluation on data collected on public roads.

When operating outdoors global positioning systems (GPS) should be the first choice for localization. In particular, RTK-GPS can give accuracy well sufficient for autonomous driving. The need for other localization arises when GPS is not available, which can happen near tall buildings or in tunnels for example. Dead-reckoning can maintain the localization for a time but will eventually drift beyond acceptable limits. There are well known methods to localize by comparing sensor readings such as from cameras or lidar to a map. The map, pose, and the sensor model together predict a sensor measurement which can then be compared to the actual one. Optimizing that match with respect to the pose gives a localization relative to the map.

For autonomous driving there are some constraints that could influence the choice of both sensor and the localization method. When it comes to the sensors it is important that the sensors be rated for automotive environments. Time to failure is critical if the system is to be both reliable and

${ }^{1}$ Erik Ward and John Folkesson are with the Centre for Autonomous Systems at KTH Royal Institute of Technology, Stockholm, SE-100 44, Sweden. $\{$ eward, johnf $\}$ akth.se

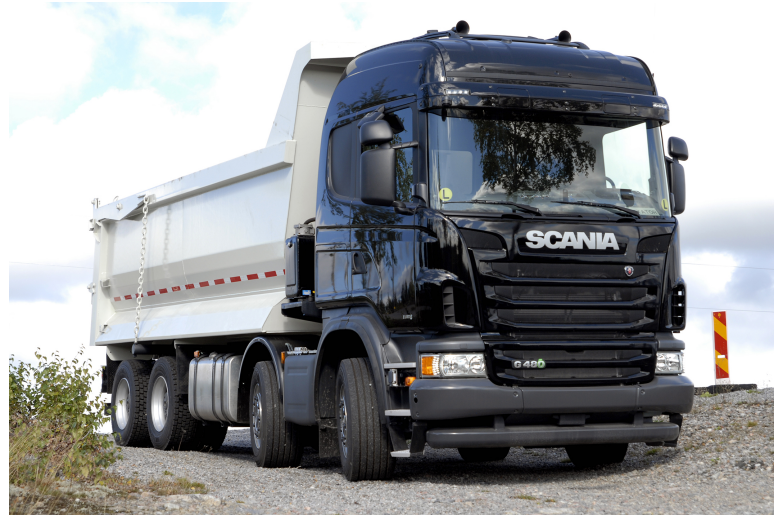

Fig. 1. Sensor equipped truck used for experiments.

economical. The size, power, mounting and computational requirements also matter. In these regards SRR provide a cheap and durable option to other sensors. Like lidar sensors, they give a bearing and a range and have the added advantage of providing Doppler based relative velocity. They are available in packages designed for automotive applications. Compared to lidar they are cheaper and have no moving parts. Compared to cameras they do not require computation of depth and are not affected by lighting or weather. The automotive SRRs used in this work where supplied as closed systems where only a limited number of already processed detections where available every time-step, rather than the original signal, which lends itself well to the application of the ICP algorithm.

The localization method requires a map and sensor model where the building and storage of the map can be thought of as a cost to be minimized. One does not need the map to contain more information than that required to predict a sensor reading from the chosen sensor from poses on the road. This is significant when one considers how many $\mathrm{km}$ of road would need to be mapped accurately for autonomous driving to be universal. For example $\mathrm{cm}$ size voxel grid over all roads would be both very large and hard to maintain as the world changes. It would also be much more than is needed. Admittedly, this does not consider other possible applications of dense 3D maps such as detection of changes. Feature based maps can be quite compact but they too require processing to build and maintain. Furthermore feature based maps often ignore much of the data that does not come from the chosen features.

Our proposal is that saved radar data from a previous recent traversal of the route provides the needed information to localize and no more. It is also easy to maintain as one 
can simply swap out the data for more fresh data when changes are detected. These make it worth investigating in the autonomous driving setting. We look at how well such an approach can localize a truck on actual roads.

The main contribution of this work is the use of ICP and EKF to localize with SSRs relative to a single previous pass in an autonomous driving scenario. This approach requires less data and less computations than previously tried methods. These are evaluated using actual data collected by an instrumented vehicle shown in fig. 1. We show that we achieve localization performance that is sufficient for most autonomous road driving applications and comparable to the most relevant prior work used as a benchmark for our results.

\section{RELATED WORK}

The use of radar in autonomous vehicle navigation is well summarized in [2]. In [3] radar scan matching SLAM is done using the Fourier-Mellin transform. They motivate this more complex matching scheme by claiming that matching using ICP will be prone to failure due to noise and the sparseness of the data. No citation is given supporting that claim. We acknowledge that these issues do present challenges and that ICP can indeed fail, as can other methods. We show here that despite this, ICP can be used successfully with radar on typical public roads.

Localization to a map is an easier problem than simultaneous localization and mapping, SLAM, but the methods and prior work on SLAM are related to localization. A feature based EKF SLAM approach using radar was shown in [4]. This is similar to our approach except for the use of strong reflections as the features. We use the entire scan with no need to extract features. This is a simplification and to be preferred if it does not worsen the localization significantly. Depending on the density of the features scan matching can potentially perform better than feature based methods.

Short range radar is used to localize in an autonomous driving context in [5]. There the focus is on modeling the dynamics in the environment by a semi-Markov process. The heart of the system is a grid map representation where every cell's dynamics are modeled as a dynamic process. This method is only sensible for localization in a limited area. The final localization is done using Monte Carlo Localization, $M C L$ ), (aka a particle filter) [6]. MCL is the preferred method of localization in robotics as it can represent multimodal distributions. It is not a practical alternative to the EKF in our case since the 'measurements' from ICP would be difficult to compute on a per particle basis.

The most relevant prior work is Lundgren et. al [7]. They used production grade automotive radars, a low-cost GPS and line marking detections from a camera system together with a detailed map to localize the vehicle. Here the map was created using an accurate RTK-GPS and by clustering the radar measurements into point sources which could be considered a kind of feature based map. Localization was performed using a particle filter where the radar measurement was modeled as a Poisson process describing the number of measurements from point sources or clutter. Classifying much of the available radar measurements as clutter and using a point source model for landmarks is an attempt to reduce computational cost and memory requirements but comes at the expense of discarding much of the available information. The same authors also used a variant of the Expectation Maximization algorithm (EM) to find a maximum a posteriori estimate of the landmark map through extensive offline computation [8]. Our method is significantly simpler than these methods and, at least on our data, performs comparably well.

Building and maintaining a radar based grid map is difficult due to the fluctuating radar cross section of the landmarks [9], [10]. Here they use millimeter wave radar where the entire return in each beam is processed allowing more than one detection per beam. They show that evidence methods (such as Dempster-Shafer) can give improvements over the more traditional Bayesian mapping approach. In [11] these authors go so far as to build models that can be used to predict radar returns. Our SSR only gives a list of the strongest returns so we can not utilize these more advanced methods. These works highlight the advantage our method has in avoiding the computation of a map.

In localization with laser scanners, scan matching using the Iterative Closest Point algorithm (ICP) [12], described in section III-A, is a popular approach that provides excellent results [13]. Automotive SRRs are less accurate than laser scanners and provide relatively sparse measurements. The standard ICP algorithm does not take into account sensor uncertainty and the effect of uncertainty of our own pose, nor does it consider the likelihood of a particular result with respect to the possible motion of the vehicle between scans. By incorporating the ICP algorithm in a Kalman filtering framework we are able to remove outlier scan matching results and provide a filtered estimate that is much smoother than that obtained by ICP alone.

Scan matching algorithms match the overlap in two sensor measurements to estimate the sensor spatial transformation (motion) between the two measurements. When used between consecutive scans this can provide a very accurate replacement to wheel-based or inertial sensor odometry. In [14] Barjenbruch et. al showed impressive results when estimating velocity and yaw-rate using a similar radar sensor to the ones used in this work. Their approach optimized both the discrepancy in position of radar detections and the Doppler velocity with respect to the vehicles motion. However, even small errors in methods which calculate the relative motion between consecutive sensor readings will accumulate when summed over a longer time. In this work we investigate how well we can match current radar detections to a map of previous radar detections to correct drift accumulated in summing relative motion estimates. The Doppler velocity of radar detections are used to discard detections originating from moving targets.

\section{POSE ESTIMATION}

We use an extended Kalman filter, EKF as our pose estimator. Although the specific vehicle model is not of 
fundamental importance to our approach we present our model here. We use a simple constant turn rate velocity model with control variables $u_{t}=(v, \omega)^{T}$ where $v$ is the speed and $\omega$ the yaw-rate:

$$
\dot{\mathbf{s}}=(\cos (\theta) v, \sin (\theta) v, \omega)^{T}+\epsilon
$$

with $\epsilon \sim \mathcal{N}(0, R)$. We formulate direct measurements, $\mathbf{z}_{\mathbf{t}}$, of the pose, $\mathbf{s}_{\mathbf{t}}=\left(x_{t}, y_{t}, \theta_{t}\right)$ along with their covariance $Q_{t}$. These are provided by a scan-matching algorithm, described in section III-A, which estimates $\mathbf{z}_{\mathbf{t}}$ such that current sensor data, described by the sensor model listed in section III-B, aligns with recorded sensor data from previous visits to the same area, stored in a map described in section III-C. The resulting localization algorithm is described in section III-D.

\section{A. Iterative Closest Point}

ICP [12], has been used extensively in localization. The algorithm works by repeating a two step procedure until convergence. The first step is matching each point in a set of source points, $X$, to the closest point in a set of reference points, $Y$, and the second step is finding the optimal transform between the source and target sets, given the assignments. Matching points by distance is a computationally efficient operation if we use a kd-tree data structure to store $Y$. Here we denote the points in $X$ as $x_{i}$ and the matched (closest) point from $x_{i}$ in $Y$ as $y_{i}$. The basic 2D ICP version optimizes the sum of squared distances between source and target points to find the rotation angle $\phi$, encoded by rotation matrix $R(\phi)$, and translation $t$.

$$
\begin{aligned}
& d_{i}=y_{i}-\left(R(\phi) x_{i}+t\right) \\
& \underset{\phi, t}{\operatorname{argmin}} \sum_{i} d_{i}^{T} d_{i}
\end{aligned}
$$

A similar optimization can be done in 3D by adding 2 angle and one translation dimensions. When the motion between scans actually is $2 \mathrm{D}$, the use of the $3 \mathrm{D}$ formulation will be less stable. When the motion has large changes to pitch or roll, the $3 \mathrm{D}$ method is needed and the reference data needs to be dense in the third dimension as well. When the motion is approximately $2 \mathrm{D}$, the $2 \mathrm{D}$ method may still be more stable even if it can not model the larger pitch and roll motions. Our data is from a 2D scan so the 3D method is not feasible. Our scenarios are therefore limited to roads that are relatively flat (not necessarily horizontal but changes in slope should be limited so that the relative motion between the reference and live scan is nearly 2D). Of road terrain for example would be a problem.

ICP can provide pose measurements for the Kalman filter by finding the optimal transformation, $(R(\phi), t)$, from the position of radar hits in the sensor frame given by our current estimate of the pose to the frame of the map. This same transformation then is applied to our current pose to give a new pose measurement.

The standard least squares formulation in eq. (2) assigns equal cost to each point and does not allow us to model the uncertainty in where measurements originate. Consider the case when our measurements are of a wall, here we would like to constrain our points to lie on the plane of the wall but not otherwise. This can be modeled probabilistically using a likelihood function where we assume points are normally distributed with a Normal distribution that corresponds to the uncertainty in where the measurement originates. For the point-to-plane case we can assign a small covariance along the plane normal and large covariance in other directions [15].

The likelihood function that we wish to optimize with respect to $R(\phi)$ and $t$ is based on the independent differences between normally distributed variables $x_{i}-y_{i}$, $x_{i} \sim \mathcal{N}\left(\mu_{i}^{X}, C_{i}^{X}\right), y_{i} \sim \mathcal{N}\left(\mu_{i}^{Y}, C_{i}^{Y}\right)$, with an optimizer:

$$
\begin{gathered}
d_{i}=\mu_{i}^{Y}-\left(R(\phi) \mu_{i}^{X}+t\right) \\
\underset{\phi, t}{\operatorname{argmin}} \sum_{i} d_{i}^{T}\left(C_{i}^{Y}+R(\phi) C_{i}^{X} R(\phi)^{T}\right)^{-1} d_{i}
\end{gathered}
$$

Using appropriate covariance matrices $C_{i}^{Y}$ and $C_{i}^{X}$ we can generalize different variants of ICP scan matching such as point-to-point, using $C_{i}^{Y}=I, C_{i}^{X}=O$, point-to-plane and plane-to-plane. For further details consult [15].

For low noise sensors such as laser scanners, extracted shapes such as lines and planes can often reliably be estimated by calculating point estimates from nearby points. Perhaps the most general approach is the Normal distribution Transform [16] which divides the space into equally sized grid cells and fits a normal distribution to encode the shape of the points in each cell. For radar data these types of shape extraction methods are very problematic since our data is corrupted by noise and clutter measurements. It is also requires the computation of the covariance matrices in eq. (3), for example: [15] performed plane-to-plane matching by extracting surface normals using principal component analysis of the 20 closest points for each matched point $x_{i}$.

We take a simpler approach using eq. (3) to model the range dependent uncertainty of the radar, as described in section III-B. We model the covariance matrices $C_{i}^{X}$ and $C_{i}^{Y}$ as isometric $C_{i}^{X} \propto I, C_{i}^{Y}=\propto I$. This also removes the dependence of the metric term $\left(C_{i}^{Y}+R(\phi) C_{i}^{X} R(\phi)^{T}\right)^{-1}$ in eq. (3) on the rotation matrix $R(\phi)$ and allows this metric matrix to be replaced by a scalar $w_{i}$. Also, points in $X$ that are more than a threshold distance $d_{\max } / \sqrt{w_{i}}$ from any point in $Y$ are not included in eq. (3).

\section{B. Sensor Model}

The radars deliver data every time-step $t$ as a list of tuples, scan $_{t}=\left[\left(r_{j}, \phi_{j}, \dot{r}_{j}, A_{j}\right), \ldots\right]$, one for each detection $j$, containing the range, $r$, the bearing to the target $\phi$, the Doppler velocity $\dot{r}$ and an amplitude value corresponding to the attenuation of the signal power, $A$.

Scan matching is performed in a global Cartesian coordinate system in which the map points are stored. We model the uncertainty of the position of our radar detections by viewing measurements as normally distributed around the current scan positions, $X$, and map positions, $Y$. As we 


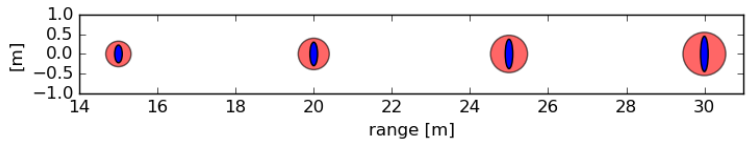

Fig. 2. Isotropic covariance, red, compared to linearized covariance, blue, for modeling radar uncertainty. The standard ICP treats all points the same, isotropic approximation models the increase in uncertainty with range and the linearized method fully models the covariance.

described in the previous section we model the distributions with isotropic covariance matrices which we further specify here as $C_{i}^{X}=\left(r_{i}^{X} \sigma_{\phi}+c\right)^{2} I, C_{i}^{Y}=\left(r_{i}^{Y} \sigma_{\phi}+c\right)^{2} I$, where $r_{i}^{X}$ is the range of the point and $\sigma_{\phi}$ the standard deviation of the bearing. Figure 2 shows the approximation we make in the radar model.

ICP provides a transformation that gives the minimum of the cost in eq. (3) but does not provide any estimate of the uncertainty in that transformation. We use the Hessian of eq. (3) at the optimum to compute an estimate of the covariance, $Q_{t}$, of the measurement of the vehicle pose used in the EKF, $z_{t}$. We calculate an approximation of $Q_{t}$ using the Hessian method as in [1]:

$$
\tilde{Q}_{t}=\frac{E_{\min }(\phi, t)}{n-3}\left(\frac{1}{2} H\right)^{-1}
$$

where $E_{\min }(\phi, t)$ is the optima of eq. (3), $H$ the Hessian and $n$ the number of points used in the numerical approximation. Under-estimation of the measurement uncertainty in the EKF can lead to divergence and brittleness in the estimates. Over-estimates have a milder consequence of smoothing the estimates which then become pessimistic. Therefore, we allow for scaling of $\tilde{Q}_{t}$ by a constant $c_{\sigma} \geq 1, Q_{t}=c_{\sigma} \tilde{Q}_{t}$, determined empirically.

When localizing we want to remove measurements from moving targets from both $X$ and $Y$. By thresholding the difference between the measured Doppler velocity $\dot{r}_{i}$ with the expected Doppler velocity for stationary targets, $V_{i}$, [14] we can remove most moving targets from consideration. $V_{i}$ is the Doppler velocity that would be measured from a stationary target based on the motion of the sensor, where the sensor pose relative to the vehicle pose, $\mathbf{s}_{t}$, is $\left(x_{s}, y_{s}, \alpha_{s}\right)^{T}$ :

$$
\begin{aligned}
V_{i}\left(u_{t}, \phi_{i}\right)= & -\left(v_{t}-\omega_{t} y_{s}\right) \cos \left(\phi_{i}+\alpha_{s}\right) \\
& -\left(\omega_{t} x_{s}\right) \sin \left(\phi_{i}+\alpha_{s}\right)
\end{aligned}
$$

\section{Map Creation}

Our map consists of the positions of radar detections in UTM coordinates according to assumed ground truth pose measurements from a RTK-GPS. Measurements are grouped into sets of 10 scans, converted to UTM coordinates, and stored together with the last pose associated with these scans. This allows us to efficiently look-up nearby map points by querying for all measurement sets within a threshold distance using a kd-tree data structure.

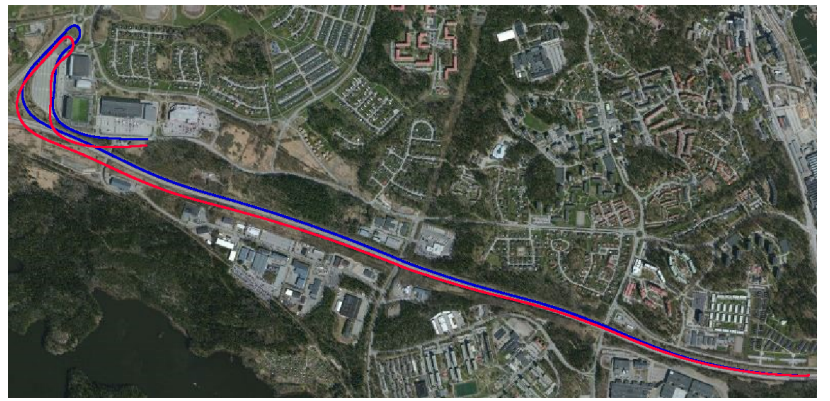

Fig. 3. Path taken during localization. Red trajectory is using odometry only, blue using proposed method. Satellite imagery is from Bing.

\section{Localization Algorithm}

Our localization algorithm is EKF recursive pose estimation using the motion and measurement models as described in previous sections. As input the algorithm takes the current pose estimate and control variables along with scan data from the radars. Reference scan data in a global coordinate system is available from the data structure map. The estimation parameters $\mu_{t}, \Sigma_{t}$ are the updated mean and covariance of the pose $\mathbf{s}_{\mathbf{t}}$.

After each motion prediction step we compare the map to current sensed data in order to generate the measurement $z_{t}$ and measurement noise $Q_{t}$ for the EKF update step. In order to have an adequate amount of data for scan-matching we store the last $k$ scans in the data structure we call a keyframe which converts the radar detections from several scans into the same coordinate system using odometry information. The keyframe acts as a FIFO queue consisting of the last $k$ scans at each time-step. We compute the best alignment of the scans stored in the keyframe with reference data using eq. (3). We then check if $z_{t}$ is within a confidence threshold from our predicted pose if not we discard the improbable ICP results as outliers.

\section{EXPERIMENTS}

We evaluated our method using two data sets, one, data 1 , that we collected and one, data 2 , provided by the authors of [7]. Data 2 allows us to directly compare our results with [7] giving use a baseline. To collect data 1 we have used the truck shown in fig. (1) which is equipped with $76 \mathrm{GHz}$ Pulse-Doppler Delphi Short Range Radar (SRR) sensors and a high precision Oxford RT2002 GPS/IMU unit. The SRRs deliver up to 64 detections each at $20 \mathrm{~Hz}$ while the Oxford RT2002 delivers measurements at $100 \mathrm{~Hz}$ but we have downsampled the speed and yaw rate signals to $20 \mathrm{~Hz}$. The two radars used are mounted on left and right side of the cabin, $66 \mathrm{~cm}$ behind the front and $50 \mathrm{~cm}$ above the ground, are directed perpendicular to the direction of travel and have a field of view of $r \in[0.5 \mathrm{~m}, 80 \mathrm{~m}], \phi \in\left[-75^{\circ}, 75^{\circ}\right]$. The measurements errors are modeled as normally distributed noise where $99 \%$ confidence levels correspond to an angle error of $\pm 2.2^{\circ}$ and a range error of $0.25 \mathrm{~m}$. The GPS/IMU unit is accurate within a few centimeters as long as the unit is connected to the internet and can receive RTK correction 


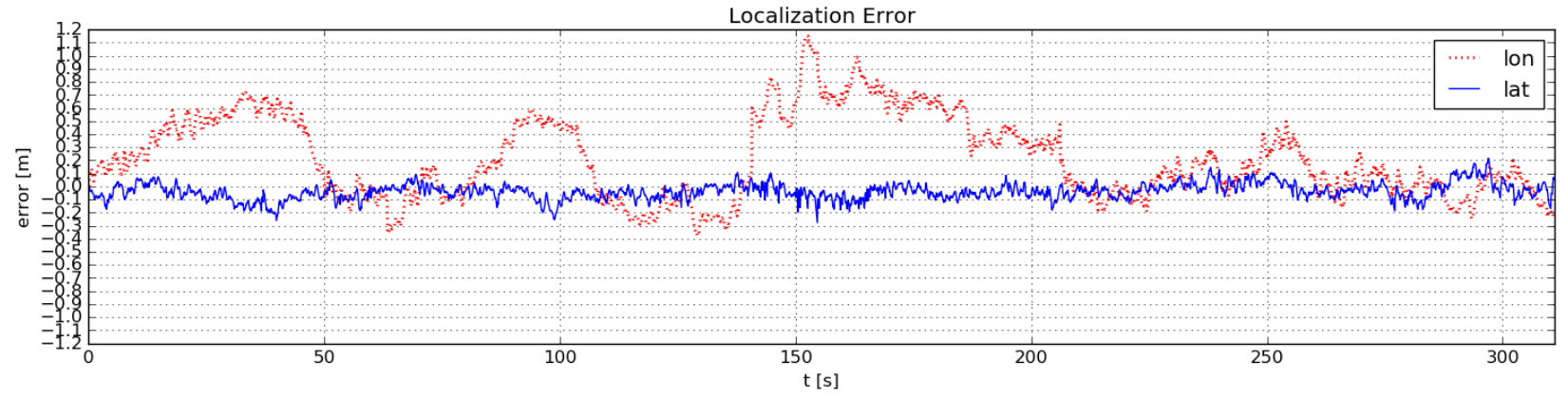

Fig. 4. Lateral (solid blue) and longitudinal (dotted red) errors in meters using proposed localization. Negative numbers correspond to left and behind, respectively.

data. We consider the pose data as ground truth when we have RTK coverage.

The truck was driven twice along the same route. The route includes both a highway section and smaller roads in a suburban setting Fig. 3. We use the radar and RTK-GPS/IMU from the first pass to create the map. The RTK-GPS/IMU from the second pass will be used as ground truth to compute the pose errors. Our control signals $v, \omega$ was taken from the Oxford sensor which gives very good speed measurements but worse yaw-rate, corresponding to a longitudinal drift of $0.02 \mathrm{~m}$ and a lateral drift of $1.2 \mathrm{~m}$ when driving straight for $100 \mathrm{~m}$. The $5.4 \mathrm{~km}$ long path for the second run is shown in figure 3 . The experiment starts on the highway, the truck then exits and heads towards a round-about after which the final stretch of the experiment takes place on a smaller road on the edge of a residential area adjacent to several large buildings.

\section{RESULTS AND DISCUSSION}

We start with data 1 that we collected ourselves. We needed to first determine the best values for the number of scans per keyframe $k$ and for the measurement covariance scale factor $c_{\sigma}$. We optimized these with respect to root mean square error and maximum error on a small training data-set, separate from our evaluation data-set, and found the best values to be: $k=4, c_{\sigma}=50.0$. Our process noise covariance was $R=\Delta t \operatorname{diag}(0.01,0.01,0.005)$, where $\Delta t$ is the time between EKF prediction steps, the ICP outlier threshold, $d_{\max }$, was set to $d_{\max }=1.0$ and for the sensor model we have used $c=0.1$.

Figure 4 shows the lateral and longitudinal errors for localizing with point-to-point ICP when compared to the Oxford IMU/GPS unit. The root mean square lateral error is $7.3 \mathrm{~cm}$ and at worst $27.8 \mathrm{~cm}$. The root mean square longitudinal error is $37.7 \mathrm{~cm}$ and at worst $115.1 \mathrm{~cm}$. These errors are somewhat exaggerated because of time synchronization issues between the sensor measurements and ground truth data of up to 0.01 seconds which would correspond to at worst a longitudinal error of $24 \mathrm{~cm}$ for the maximum speed of $24.1 \mathrm{~m} / \mathrm{s}$ during localization. During the experiment there where four large RTK-GPS corrections, that essentially caused the ground truth reference position to shift much more
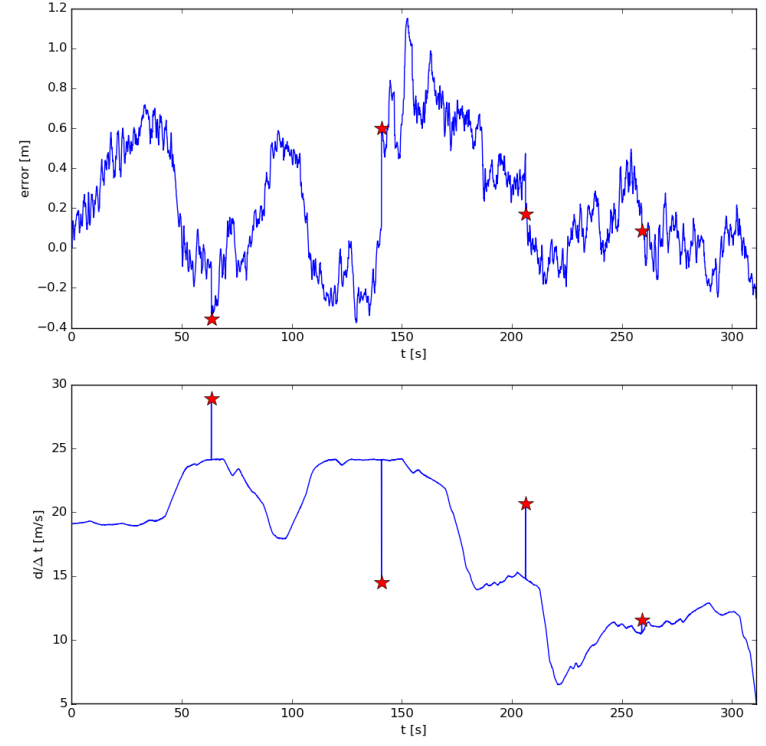

Fig. 5. Longitudinal error, same as in fig. 4, top and displacement per measurement of the RTK-GPS divided by the time difference of measurements, bottom.

that the speed of the vehicle would suggest. These correspond to some sharp edges in the error in fig. 4 which can be clearly seen in fig. 5 where we plot the reported displacement of the RTK-GPS per time-step, $d$, divided by the difference in time of its measurements, $\Delta t$. Here large changes in $d / \Delta t$ are marked with red stars.

Since the truck was driven along a highway in the first part of the localization experiment, there are often few objects to detect besides guard rails and vegetation such as trees and bushes growing parallel to the highway which mostly corrects the lateral position of the vehicle. This allows small longitudinal errors of the ICP algorithm to grow over time until something that can fix the longitudinal position is detected, which can be seen in fig. 4 . During some segments along the highway there are more distinct objects such as adjacent buildings which allows the algorithm to correct the longitudinal position, giving rise to the sharp decrease in longitudinal errors after a period of increase. After around 


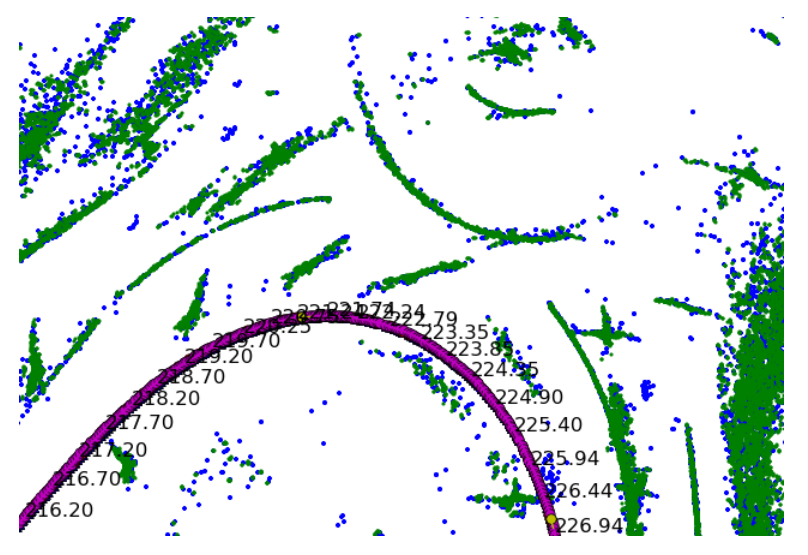

Fig. 6. The ICP global position measurements are shown in purple for inliers and in yellow for outliers (only a few outliers at the right most end), during a right turn near a round-about. Current radar detections after ICP shown in green and map data shown in blue. Experiment time shown as black text.

200 seconds the truck enters an area with a round-about, here there are many curbs to detect for the sensors which helps correct the position. The resulting ICP measurements for the EKF are shown in fig. (6). After this area the truck enters a smaller road where there are several adjacent buildings, which also provides detections that can correct longitudinal errors. The localization performance is mostly dependent on the quality of discernible features available such as corners of buildings or detected curbs with a specific shape, such as near the roundabout, rather than the speed of the vehicle or how fast it is turning.

In [7] the localization performance was evaluated with respect to a maximum allowed lateral error of $20 \mathrm{~cm}$ and longitudinal error of $100 \mathrm{~cm}$. We show our results using this same criteria in the first row of table I.

TABLE I

COMPARISON OF TIME WITHIN ERROR LIMITS

\begin{tabular}{r|c|c} 
Method & Lateral & Longitudinal \\
\hline Our method on Data 1 & $99 \%$ & $99 \%$ \\
Our method on Data 2 & $30 \%$ & $89 \%$ \\
[7] using radar on Data 2 & $23 \%$ & $90 \%$ \\
[7] radar and cameras on Data 2 & $87 \%$ & $94 \%$
\end{tabular}

For a direct comparison, we have evaluated our method data 2 which is that same as used in [7]. It includes detections from a single radar mounted on the grill of a car, speed and yaw-rate from a speedometer and a gyroscope and a RTKGPS for reference. The car is also equipped with a low cost GPS and a camera that was used to detect lane markings. The data-set is divided into two parts where the same road is driven in each part.

This data-set is significantly more challenging than the previous data-set, not only is there only one radar instead of two, but the sensor system was configured to filter out many of the detections, leading to a far more sparse data-set, where mostly guard rails, delineator posts and traffic signs where detected and not much of the surrounding terrain. This filtering was likely of benefit to the method in [7]
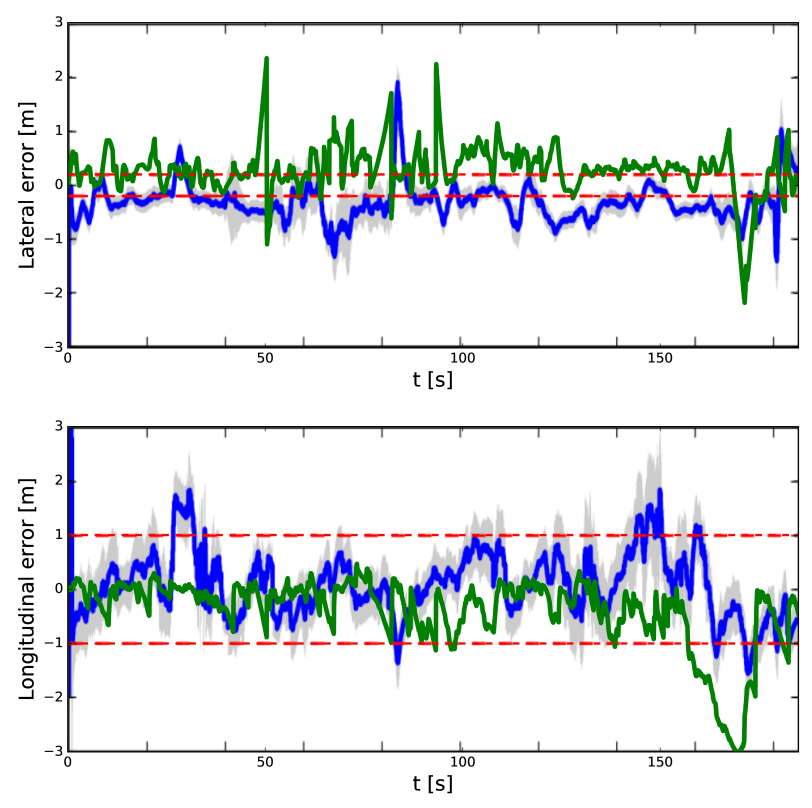

Fig. 7. Comparison of our proposed method, green, overlaid on the plot from [7], blue, on the same data using the same sensors.

but our method could have used the data that was filtered out. Furthermore, the radar data has larger inconsistencies between the mapping run and the evaluation run than in the truck experiment.

For this second data-set we used slightly different parameters from the truck data-set, the process noise $R$ was larger to account for less accurate odometry information than used in the truck experiment, $R=\Delta t \operatorname{diag}(0.05,0.05,0.05)$, $d_{\max }$ was set to $d_{\max }=10.0$ to allow for a larger search of correspondences in the sparse data-set. One difference between the results from Lundgren et. al and ours is that they start their initial estimate at the first low cost GPS measurement and we start at the assumed known ground truth position. This allowed us to set $d_{\max }$ lower than if we needed to do the initial convergence. We show our results on this data in the second row of table I. Also shown are the numbers from Lundgren et. al.

The low number of radar detections in this data-set leads to sections of the evaluation run where there are no matching data so that the ICP algorithm cannot be performed and here localization relies on odometry alone. This necessitates the large value for $d_{\max }$ since we need to search a larger area for correspondences after such sections. This in turn, adds incorrect correspondences for the ICP algorithm which leads to many erroneous ICP results which necessitates outlier removal and the use of a Kalman Filter to smooth the estimate. For example during the large spike in lateral error in fig. (7) error at time 47-50 seconds there are only two radar detections. This is not enough to perform ICP, the same phenomenon occurs at 76-82 seconds. The peak in error at 94 seconds occurs after a section with no radar data where the estimated pose covariance has grown large and our confidence based outlier removal is unable to filter out a few 
bad ICP results. At around 158 seconds the filter locks on to the wrong longitudinal position, this is during a section of two-lane road with a guard rail separating the two lanes. During the mapping run, the car drove on the other lane of the road which causes substantial inconsistencies between the radar data for the evaluation run and the mapping run. After this the guard rail separating the lanes disappear and the car enters a turn. Here our method has substantial problems in correcting the position estimate.

Despite the data being filtered for the feature based mapping method we were nevertheless able to localize about as well as the more complex feature based method. It is hard to draw strong conclusions from this short comparison. Comparing to the unfiltered longer data set on public roads that we collected ourselves we can see that our method does benefit greatly from more dense data.

\section{CONCLUSIONS}

In this paper we have presented a localization algorithm for a radar equipped vehicle based on an EKF using ICP scan matching against a recorded map of previous sensor data from a single pass through the same route. We show that this can produce good localization of an autonomous vehicle by evaluating with actual radar data collected on public roads. This method has the advantage of not requiring large amounts of mapping data to be processed offline but rather can use the most recent data from a single pass along the same route with the same sensors.

\section{ACKNOWLEDGMENT}

We gratefully acknowledge that this work was carried out within the iQMatic - future autonomous transport systems, VINNOVA funded project, number 2012-04626. We would also like to thank the Autonomous Transport Solutions team at Scania for the use of their experimental platform and help with collecting the data. We are also grateful to the authors of [7] for providing the data for comparison to their work.

\section{REFERENCES}

[1] O. Bengtsson and A.-J. Baerveldt, "Robot localization based on scanmatching - estimating the covariance matrix for the idc algorithm," Robotics and Autonomous Systems, vol. 44, no. 1, pp. 29-40, 2003.
[2] M. Adams, J. Mullane, and E. Jose, Robotic navigation and mapping with radar. Artech House, 2012.

[3] P. Checchin, F. Grossier, C. Blanc, R. Chapuis, and L. Trassoudaine, "Radar scan matching slam using the fourier-mellin transform," in Field and Service Robotics, ser. Springer Tracts in Advanced Robotics. Springer Berlin Heidelberg, 2010, vol. 62, pp. 151-161.

[4] M. Dissanayake, P. Newman, S. Clark, H. F. Durrant-Whyte, and M. Csorba, "A solution to the simultaneous localization and map building (slam) problem," Robotics and Automation, IEEE Transactions on, vol. 17, no. 3, pp. 229-241, 2001.

[5] M. Rapp, M. Hahn, T. Markus, J. Dickmann, and K. Dietmeyer, "Semi-markov process based localization using radar in dynamic environments," in Intelligent Transportation Systems (ITSC), 2015 IEEE 18th International Conference on. IEEE, 2015, pp. 423-429.

[6] S. Thrun, D. Fox, W. Burgard, and F. Dellaert, "Robust monte carlo localization for mobile robots," Artificial Intelligence, vol. 128, no. 12, pp. 99 - 141, 2001. [Online]. Available: http://www.sciencedirect.com/science/article/pii/S0004370201000698

[7] M. Lundgren, E. Stenborg, L. Svensson, and L. Hammarstrand, "Vehicle self-localization using off-the-shelf sensors and a detailed map," in Intelligent Vehicles Symposium Proceedings, 2014 IEEE. IEEE, 2014, pp. 522-528.

[8] M. Lundgren, L. Svensson, and L. Hammarstrand, "Variational bayesian expectation maximization for radar map estimation," Signal Processing, IEEE Transactions on, vol. PP, no. 99, pp. 1-1, 2015.

[9] J. Mullane, M. Adams, and W. Wijesoma, "Evidential versus bayesian estimation for radar map building," in Control, Automation, Robotics and Vision, 2006. ICARCV '06. 9th International Conference on, Dec 2006, pp. 1-8.

[10] J. Mullane, E. Jose, M. D. Adams, and W. S. Wijesoma, "Including probabilistic target detection attributes into map representations," Robotics and Autonomous Systems, vol. 55, no. 1, pp. 72-85, 2007.

[11] E. Jose, M. Adams, J. Mullane, and N. Patrikalakis, "Predicting millimeter wave radar spectra for autonomous navigation," Sensors Journal, IEEE, vol. 10, no. 5, pp. 960-971, May 2010.

[12] F. Lu and E. Milios, "Robot pose estimation in unknown environments by matching $2 \mathrm{~d}$ range scans," Journal of Intelligent and Robotic Systems, vol. 18, no. 3, pp. 249-275, 1997.

[13] K. Yoneda, C. Yang, S. Mita, T. Okuya, and K. Muto, "Urban road localization by using multiple layer map matching and line segment matching," in Intelligent Vehicles Symposium (IV), 2015 IEEE. IEEE, 2015, pp. 525-530.

[14] M. Barjenbruch, D. Kellner, J. Klappstein, J. Dickmann, and K. Dietmayer, "Joint spatial-and doppler-based ego-motion estimation for automotive radars," in Intelligent Vehicles Symposium (IV), 2015 IEEE. IEEE, 2015, pp. 839-844.

[15] A. Segal, D. Haehnel, and S. Thrun, "Generalized-icp." in Robotics: Science and Systems, vol. 2, no. 4, 2009.

[16] P. Biber and W. Straßer, "The normal distributions transform: A new approach to laser scan matching," in Intelligent Robots and Systems, 2003.(IROS 2003). Proceedings. 2003 IEEE/RSJ International Conference on, vol. 3. IEEE, 2003, pp. 2743-2748. 